\title{
Ações de reabilitação de pessoas com deficiência na estratégia da saúde da família da Fundação Zerbini e Secretaria Municipal de Saúde de São Paulo - Região Sudeste - Sapopemba/Vila Prudente - período 2000/2006*
}

\author{
Rehabilitation actions for disabled people in the \\ strategy of family health of the Zerbini Foundation \\ and the Municipal Health Department of São \\ Paulo - Southeast Region - Sapopemba/Vila \\ Prudente - period 2000/2006
}

\author{
Eucenir Fredini Rocha ${ }^{1}$, Márcia Regina Kretzer ${ }^{2}$
}

\begin{abstract}
ROCHA, E. F.; KRETZER, M. R. Ações de reabilitação de pessoas com deficiência na estratégia da saúde da família da Fundação Zerbini e Secretaria Municipal de Saúde de São Paulo - Região Sudeste - Sapopemba/Vila Prudente - período 2000/2006. Rev. Ter. Ocup. Univ. São Paulo, v. 20, n. 1, p. 59-67, jan./abr. 2009.

RESUMO: O objetivo do artigo é descrever a implantação das ações de reabilitação na Estratégia da Saúde da Família da Região de Sapopemba/Vila Prudente - Fundação Zerbini/Secretaria Municipal de Saúde de São Paulo, no período de 2000-2006. As fontes de pesquisa foram os documentos de registros da experiência: o projeto de implantação (2000), os relatórios de avaliação (2001, 2003 2008), anotações de reuniões da equipe de reabilitação (2000-2006), os planejamentos semestrais e anuais, as planilhas de registro das atividades diárias dos profissionais de fisioterapia, fonoaudiologia e terapia ocupacional e dos atendimentos dos usuários dos serviços (2000-2006). Como resultados pode-se visualizar: 1 . as estratégias utilizadas na implantação da proposta e os desafios enfrentados; 2 . a rotina estabelecida pela equipe de reabilitação junto às equipes de saúde da família, de saúde mental e saúde bucal; 3. o perfil da população atendida e os critérios de elegibilidade; 4. as modalidades assistenciais desenvolvidas. Os dados possibilitaram compreensão da constituição das atividades de reabilitação na Estratégia da Saúde da Família, as atribuições dessas equipes matriciais e as rupturas ético-epistemológicas e institucionais com o modelo tradicional de reabilitação.
\end{abstract}

DESCRITORES: Saúde da família. Reabilitação. Atenção primária à saúde. Modalidade de fisioterapia. Fonoaudiologia. Terapia ocupacional.

\footnotetext{
* Este trabalho é parte dos resultados de pesquisa financiada pelo CNPq - Conselho Nacional de Desenvolvimento Científico e Tecnológico

- Edital MCT-CNPq / MS-DAB/SAS - No 49/2005 - Processo 202234/2005-7.

1. Profa. Dra. do Depto. de Fisioterapia, Fonoaudiologia e Terapia Ocupacional da Faculdade de Medicina da USP. E-mail: eucenir@usp. br

2. Enfermeira, Doutoranda em Saúde Coletiva da Universidade Federal de São Paulo. E-mail: mrkretzer@terra.com.br
} 


\section{APRESENTAÇÃO}

atenção à saúde e reabilitação das pessoas com
deficiências e incapacidades está prevista no
SUS, nos diferentes níveis do sistema de saúde, desde 1993 (BRASIL, 1993), no entanto, a assistência a essa população encontra-se, predominantemente, vinculada às ações de nível secundário ou terciário, constituídas através de atendimentos especializados por patologias, localizados nos centros urbanos e ligados, em sua maioria, à filantropia ou a centros universitários.

Essa modalidade de organização de serviços tem gerado problemas no acesso, porque muitos desses usuários têm dificuldades na locomoção e na acessibilidade ao transporte público e aos equipamentos de saúde, impondo limites objetivos à integralidade da assistência a esses usuários (ROCHA; KRETZER, 2008).

Acesso, integralidade, humanização, equidade são temas constantes na bibliografia científica que trata da organização e qualidade dos serviços de saúde no SUS, a especialização extremada das intervenções e a hierarquização dos serviços são consideradas por vários autores como fatores que contribuem para limitar as possibilidades de universalidade do SUS. Nessa pauta, a Estratégia da Saúde da Família' (ESF) emerge como uma proposta assistencial que pode possibilitar a reversão do modelo vigente de atenção à saúde da população.

Somente em janeiro de 2008 que a proposta de ações de reabilitação na ESF foi contemplada através da Portaria no. 154 (BRASIL, 2008) que criou os Núcleos de Apoio à Saúde da Família - NASFs. O objetivo dos NASFs é ampliar o escopo das ações da Atenção Básica (AB) e devem ser constituídos por profissionais de diferentes áreas de conhecimento, inseridos em núcleos de ação para atuar em parceria com as equipes de saúde da família, compartilhando as práticas em saúde nos territórios adstritos.

As Ações de Reabilitação constituem um dos núcleos a ser desenvolvido pelos NASFs e devem promover a redução das incapacidades e deficiências a fim de melhorar a qualidade de vida dos indivíduos e favorecer a sua reinserção social, combatendo a discriminação e ampliando o acesso ao sistema de saúde (BRASIL, 2008). A implantação dos NASFs está, atualmente, em construção em muitos municípios do país.

É somente da década de 90 para cá, que a inserção de propostas de atenção à saúde da pessoa com deficiência e incapacidades na AB/ESF está se tornando uma realidade no Brasil. Muitas dessas ações foram e estão em desenvolvimento, filiadas a projetos didático-assistenciais de cursos universitários de fisioterapia, fonoaudiologia e terapia ocupacional, em sua maioria nos estados de São Paulo, Minas Gerais, Paraná e Ceará ${ }^{2}$ (ROCHA; KRETZER, 2008).

As únicas experiências descritas na literatura que dão notícias de equipes de reabilitação compostas por diferentes categorias profissionais inseridas na $\mathrm{AB} / \mathrm{ESF}$ e empreendidas por gestores públicos são: 1. Camaragibe/ PE/Secretaria Municipal de Saúde (SMS) (2000/2005); 2. Núcleos de Reabilitação em Belo Horizonte/MG/SMS (2004/2008); 3. São Paulo/SP, através da Secretaria Estadual de Saúde/SP (SES\SP) em parceria com ESF/Fundação Zerbini (FZ) e ESF/Casa de Saúde Santa Marcelina (CSSM), nas regiões Sudeste e Leste do município $(2000 / 2008)^{3}$ (BOURGET, 2008; ROCHA; KRETZER, 2008).

Este artigo apresenta a experiência de implantação das ações de reabilitação na ESF/Fundação Zerbini da Secretaria Municipal de Saúde de São Paulo (SMS/SP), no período de 2000-2006 na região de Sapopemba e Vila Prudente. Analisa o modelo assistencial desenvolvido e aponta os deslocamentos epistemológicos produzidos por essa proposta e demonstra que as ações de reabilitação na $\mathrm{AB} / \mathrm{ESF}$ facilitam o acesso, a integralidade, a equidade e humanização dos serviços de saúde. Por fim, assinala também as implicações práticas de organização das atividades das equipes de reabilitação e do cotidiano institucional com a pretensão de contribuir para a reprodução desse modelo assistencial.

Os dados aqui apresentados são parte dos resultados da pesquisa de "Avaliação da Implantação das Ações de Reabilitação no Programa da Saúde da Família (PSF) da Fundação Zerbini e Secretaria Municipal de Saúde de São Paulo - Região Sudeste - Sapopemba/Vila Prudente periodo 2000/2006".

Foram considerados como fonte de informação e objeto empírico da pesquisa os seguintes documentos: $1^{\circ}$ ) registros da experiência, que compreendem o projeto de

1. Inicialmente denominado de Programa da Saúde da Família (PSF).

2. As experiências do Paraná e Ceará foram organizadas exclusivamente no contexto de projetos didático-assistenciais da graduação ou pós-graduação, como residências multiprofissionais.

3. A ESF da Fundação Zerbini teve como parceiro administrativo, primeiramente, a SES/SP, com o processo de municipalização em São Paulo, em 2003, a Secretaria Municipal de Saúde de São Paulo (SMS/SP) assumiu as responsabilidades técnicas sobre o projeto. 
implantação (2000), os relatórios de avaliação (2001, 2003, 2008), as anotações de reuniões da equipe de reabilitação (2000-2006), os planejamentos semestrais e anuais, as planilhas de registro das atividades diárias dos profissionais de fisioterapia, fonoaudiologia e terapia ocupacional e dos atendimentos dos usuários (2000-2006) e $2^{\circ}$ ) os textos da literatura nacional sobre a inserção de ações de reabilitação na $\mathrm{AB} / \mathrm{ESF}$.

Narrativa e análise da implantação da atenção à saúde e reabilitação da pessoa com deficiência na ESF/FZ - Zona Sudeste do município de São Paulo

No período de 1992-2000, com a implantação do PAS (Programa de Atenção à Saúde) na cidade de São Paulo foi empreendido o desmonte do sistema de saúde pública e a ESF só pode ser implantada em meados da década de 90, através da parceria entre o Ministério da Saúde (MS), a SES/SP, FZ e CSSM. A ESF foi então denominada de Programa da Saúde da Família/QUALIS - Qualidade Integral de Saúde.

Em 2001, a gestão dos serviços de saúde do município de São Paulo retorna ao setor público e com a municipalização da administração o PSF/QUALIS/FZ tornou-se responsabilidade da SMS e passou a ser designado Programa Saúde da Família/Fundação Zerbini (PSF/FZ).

Desde a implantação do PSF/FZ e da CSSM, em meados da década de 90 , as equipes de saúde da família já contavam com o apoio de equipes de saúde mental, saúde bucal, ambulatório de especialidades e casa de parto. Essas equipes atendiam a população adstrita aos territórios abrangidos pelas equipes de saúde de família desses dois parceiros administrativos da SMS.

O aprimoramento do trabalho das equipes de saúde da família no território evidenciou a grande quantidade de pessoas com deficiências e incapacidades que necessitavam de atendimento. Em 2000 foram contratadas duas equipes de reabilitação compostas por fisioterapeutas, fonoaudiólogos e terapeutas ocupacionais para o atendimento desses usuários ${ }^{4}$.

Embora, durante os anos de 2000\01, a implantação da proposta tenha ocorrido nas duas regiões com processos semelhantes, aqui será narrada somente a experiência do PSF/FZ, em Sapopemba e Vila Prudente, região sudeste do município de São Paulo.
Para elaboração do projeto, a SES/SP convidou uma técnica da FZ e o REATA - Laboratório de Estudos em Reabilitação e Tecnologia Assistiva da FMUSP. No decorrer do processo de implantação, a técnica tornou-se coordenadora da equipe de reabilitação $(2000 / 2007)$ e a assessoria técnica ficou com o REATA (2000/2001).

Em 2001, o PSF/QUALIS/FZ abrangia cerca de 171.600 habitantes através do trabalho de 39 equipes de saúde da família em dez Unidades Básicas de Saúde (UBS).

No processo de implantação houve muitas resistências por parte dos gestores, dos técnicos e docentes vinculados aos serviços tradicionais de reabilitação do município de São Paulo, que defendiam o gerenciamento das ações de reabilitação apartado da direção do PSF. A SES/SP coordenou a implementação da proposta e defendeu a inserção das ações de reabilitação nas equipes de saúde da família conforme previsto no projeto.

\section{Estratégias utilizadas na implantação das ações de reabilitação no PSF}

As estratégias utilizadas na implementação das ações de reabilitação no PSF tomou como ponto de partida a resistência dos próprios sujeitos envolvidos e selecionou, a partir de critérios epistêmicos e intencionalidades éticas, as atividades descritas e analisadas abaixo:

\section{Sensibilização das equipes de saúde da família e da comunidade}

Primeira fase do trabalho realizou a meta de introduzir no cotidiano dos profissionais das equipes de saúde da família e usuários temas como deficiência, incapacidade, reabilitação, inclusão e exclusão social, preconceito, entre outros. Foram utilizados diferentes recursos como atividades psico-pedagógicas, psicodramáticas, teatro de bonecos e debates. Essas atividades foram coordenadas pela SES/ SP e aplicadas por pessoal especializado. Participaram todas as equipes de saúde da família e diversos grupos da comunidade local. Cada encontro durou 8 horas e contou com a participação de cerca de 50 pessoas. A proposta atingiu 2260 profissionais do PSF/FZ e da CSSM e cerca de 1500 pessoas da comunidade, entre elas, muitas pessoas com deficiências e seus familiares, além de lideranças locais e interessadas no tema.

\footnotetext{
4. Para a CSSM foram contratdos 14 profissionais de reabilitação/89 equipes de saúde da família/30 horas/semanais cada/420 horas semanais e para a FZ foram contratados 9 profissionais de reabilitação/39 equipes de saúde da família/30 horas/semanais cada/270 horas/semanais.
} 
Com as equipes da saúde da família o tema foi abordado através de simulações de questões técnicas advindas do cotidiano institucional, como discussão de caso, possíveis diagnósticos e intervenções. Enquanto para a comunidade a opção foi por um trabalho lúdico através da técnica de teatro de bonecos, com personagens com e sem deficiências que interagiam com o público.

Os documentos de avaliação da implantação do projeto demonstram que num primeiro momento os participantes das equipes de saúde da família tiveram dificuldades em se envolver com os temas abordados. Os profissionais de nível universitário foram os mais resistentes, enquanto os grupos da comunidade mostraram maior interesse e menos preconceitos. Os relatos posteriores dos profissionais das equipes de saúde da família narram maior adesão às ações de reabilitação na AB/ESF após as atividades de sensibilização.

\section{Capacitação técnica das equipes de saúde da família e da equipe de reabilitação}

A capacitação ocorreu no período de fevereiro a novembro de 2001 e cada equipe de saúde da família teve 24 horas/aula, enquanto a equipe de reabilitação cursou 198 horas/aula.

Os temas trabalhados com as equipes de saúde da família foram: 1. Modelos Assistenciais em Reabilitação e Reabilitação Baseada na Comunidade - RBC: Estratégias de ação na comunidade e no PSF; 2. Deficiência, Incapacidade e Desvantagens. Conceitos e classificação da CIDID/ Classificação Internacional de Deficiências, Incapacidades e Desvantagem e suas relações com a CID-10/ Classificação Internacional de Doenças, ambas da OMS e os instrumentos na detecção de deficiências e intervenção na comunidade; 3. Principais quadros de deficiência e suas causas. Sinais de Alerta. Possibilidade de intervenção: orientação e encaminhamento; 4. Atenção à Saúde da Pessoa com Deficiência: o modelo proposto pelo PSF, a participação das equipes de saúde da família, da saúde bucal, saúde mental e comunidade e o papel de cada profissional de reabilitação no PSF.

Para os profissionais das equipes de reabilitação o curso abordou os seguintes pontos: 1. Violência, Preconceito, Ética e Tolerância; 2. Modelos Assistenciais em Saúde e Reabilitação; 3. Noções de Planejamento e Avaliação; 4. Estratégias de intervenção através da Territorialização; 5.
Follow up da criança de risco; 6. Técnicas de Manipulação Corporal junto à pacientes neurológicos (AVC, $\mathrm{PC}$ e outros); 7.Comunicação Alternativa e Sistemas Suplementares de Comunicação; 8. Tecnologia Assistiva, Adaptações e equipamentos de auxílio; 9 . Concessão de órteses e próteses no SUS; 10. Trabalho com a Família; 11. Conceitos e dinâmicas de trabalho em grupo; 12. Terceira Idade, os principais problemas e estratégias de intervenção; 13. Protetização dos pacientes amputados; 14. Intervenção junto à pacientes com problemas perceptivos e sensoriais; 15. Intervenção junto ao Deficiente Visual; 16. Intervenção junto ao Deficiente Auditivo; 17. Modelo de atenção à saúde do PSF: implicações para a Reabilitação; 18. Roteiros de Avaliação.

Os cursos foram eventos importantes, pois possibilitaram trocas de experiências entre os profissionais, debates sobre interdisciplinaridade na equipe de reabilitação e a constatação da necessidade de ações intersetoriais.

\section{O estudo de prevalência de deficiências e incapacidades e planejamento das ações reabilitacionais no território}

Consistiu no dimensionamento da problemática das pessoas com deficiência e/ou incapacidades no território e subsidiou o planejamento das atividades reabilitacionais em cada equipe de saúde da família. Essa atividade só foi realizada de maneira completa no PSF/FZ.

O estudo tomou como referência conceitual a CIDID ${ }^{5}$ e como referência empírica os questionários elaborados pelo REATA e coordenação técnica da equipe de reabilitação e foi aplicado pelos Agentes Comunitários de Saúde (ACSs) em todos os domicílios, por volta de 4.400 famílias, cobertos pelas dez UBSs, Foram impressas 17.000 fichas, correspondente a $10 \%$ da população local $(171.600$ habitantes).

O manuseio do questionário ocorreu em dois momentos: $1^{\circ}$ ) aplicação dos questionário em cada domicilio, onde a família respondia se havia alguma pessoa na casa com deficiência ou incapacidades e $2^{\circ}$.) os questionários aplicados foram discutidos nas reuniões das equipes de saúde da família, onde profissionais de reabilitação validavam ou não as suspeitas de deficiência ou incapacidades por meio de critérios técnicos (ROCHA et al., 2004).

5. A CIDID/OMS foi substituída em 2003 pela CIF- Classificação Internacional de Funcionalidade, Incapacidade e Saúde, que é mais abrangente e aborda temas como o desempenho nas atividades, participação social e a presença de fatores facilitadores ou não da funcionalidade e participação. 
Até novembro de 2002 foram detectadas pelos questionários 4476 pessoas suspeitas de possuírem alguma deficiência e/ou incapacidade, 2315 casos foram avaliados pela equipe de reabilitação e 1893 passaram a ser assistidos pelos profissionais das equipes, o que, conforme os registros, constituíram 4.593 procedimentos reabilitacionais no mesmo período.

O resultado do levantamento permitiu sistematizar as reuniões das equipes de saúde da família com os profissionais de reabilitação e proporcionou o amadurecimento de várias habilidades técnicas na deteç̧ão precoce da população que necessitava de ações de reabilitação.

Essa atividade revelou-se como um bom instrumento de sensibilização, capacitação e facilitadora de práticas interdisciplinares, além de contribuir para o conhecimento de forma mais detalhada das necessidades de saúde do território.

A dinâmica de discutir "casos" de pessoas com deficiências e incapacidades nas equipes de saúde da família se tornou uma rotina e como resultado dessa prática até 2006 a equipe de reabilitação realizou cerca 16.388 procedimentos reabilitacionais, demonstrando a expansão de suas ações.

Definição das atribuições e rotina do trabalho da equipe de reabilitação na $E S F$

O projeto de implementação já previa a formação de duplas dos profissionais de reabilitação que seriam referência para cerca de 8 a 10 equipes de saúde da família. A idealização da proposta de duplas objetivava a efetivação de um trabalho com características interdisciplinares no campo da fisioterapia, fonoaudiologia e terapia ocupacional.

Como o número de profissionais da equipe de reabilitação era ímpar, nove terapeutas de três categorias profissionais diferentes, foram agrupados em três duplas e um trio. Com o passar do tempo observou-se que o trio oferecia uma melhor articulação entre as diferentes áreas de conhecimento e, em 2005, a equipe foi reorganizada em três trios compostos por um fisioterapeuta, um fonoaudiólogo e um terapeuta ocupacional, que se tornaram referência para 13 equipes de saúde da família. Todavia, entre 2000-2006, com a expansão da população no território de abrangência, o número de famílias assistidas por equipe saltou de 1000 para 1600 e o trabalho ficou comprometido pelo aumento do número de usuários.

Como atribuições específicas da equipe de reabilitação na ESF, o projeto definia:

a. planejar e executar ações de intervenções em reabilitação adequadas às necessidades do território considerando as principais condições de risco de cada área/micro-área, e o mapeamento censitário das pessoas com deficiência ou incapacidade;

b. introduzir no estudo do território as necessidades específicas das pessoas com deficiências, como o acesso a logradouros públicos, transporte adaptados, escolas inclusivas etc.;

c. construir parcerias com a comunidade e organizações civis e/ou públicas visando à atenção integral;

d. realizar atendimento clínico a nível ambulatorial e domiciliar;

e. elaborar propostas e encaminhar ações com vistas a redução das condições de risco e vulnerabilidade social como a segregação e a exclusão social das pessoas com deficiências e/ou incapacidades;

f. apoiar e capacitar, de forma continua, as equipes de saúde da família e equipes de apoio nos temas específicos de reabilitação;

g. criar mecanismos permanentes de sensibilização dos profissionais das equipes e comunidade;

h. criar um sistema de registro de informações das necessidades das pessoas com deficiências e/ou incapacidades dentro dos protocolos das equipes de saúde da família;

i. realizar procedimentos de Tecnologia Assistiva/ Apoio, sugerir e acompanhar adaptações de mobiliário e de espaços nos domicílios e escolas com os recursos disponíveis na comunidade;

j. fomentar a discussão de casos de pessoas com deficiência junto às equipes de saúde da família.

Como é característico da cotidianidade, todo grupo de trabalho vai se organizando em torno de uma rotina que garante as interações subjetivas do processo de trabalho, normalmente essa rotina é fruto da espontaneidade pragmática do cotidiano. Na experiência aqui relatada a formação da rotina foi construída fundamentalmente a partir das intencionalidades descritas acima nas atribuições da equipe de reabilitação, por meio de reuniões de planejamento, discussões técnicas e operacionais, com a coordenação técnica e assessoria, onde eram socializadas e avaliadas as atividades da semana. Concomitantemente (2002-2003) ocorria a aplicação dos questionários de levantamento das pessoas com deficiência e/ou incapacidades existentes no território, que subsidiou as reuniões dos profissionais de reabilitação com as equipes de saúde da família.

A partir de 2003 foi se organizando uma rotina semanal de encontro da equipe de reabilitação para a discussão e planejamento das ações específicas, estudo da demanda e estabelecimento de estratégias de implementação de ações reabilitacionais no território.

Os profissionais da reabilitação também participavam 
de reuniões intersetoriais e atividades globais das UBSs, como campanhas de vacinação, prevenção e combate à dengue, papanicolau etc. Essas ações proporcionaram uma maior integração entre profissionais de diferentes equipes, deslocando a intervenção do eixo especializado para uma ação fundada em práticas multidisciplinares e generalistas em reabilitação.

Os atendimentos reabilitacionais ocorreram em sua maioria nas UBSs, nos domicílios e em espaços comunitários mais próximos das residências, facilitando o deslocamento do usuário e o acesso ao serviço de saúde.

Ao contrário das propostas tradicionais de reabilitação onde os usuários estão sob a tutela exclusiva das equipes especializadas, o eixo do atendimento reabilitacional na ESF/FZ ficou sob a responsabilidade das equipes de saúde da família e dos profissionais de reabilitação de referência dessas equipes, para promover a assistência integral à saúde e não apenas o atendimento exclusivo às demandas decorrentes dos processos de deficiência e incapacidades.

A integralidade na atenção à saúde só se intensificou no segundo ano de implantação com a efetivação de trabalhos conjuntos com as outras equipes de apoio (bucal, mental e especialidades), como por exemplo, a capacitação das equipes de saúde bucal para o atendimento de pessoas com deficiências ou a constituição de grupos de atividades em parceria com a saúde mental, como a criação de brinquedotecas e grupos de familiares e crianças em situação de vulnerabilidade.

A demanda de cada território e a necessidade de uma maior resolutividade e integralidade na assistência (ROCHA; KRETZER, 2008) nortearam a organização das atribuições acima descritas em atividades e procedimentos que podem ser classificados da seguinte forma:

- Cuidados terapêuticos: compreende as estratégias de intervenções terapêuticas que proporcionam acesso do usuário aos cuidados de saúde, para isso foram organizados terapias individuais/consultas, atendimentos em grupo, atendimentos domiciliares, adaptações ou confecções de objetos para atividades da vida diária e prática, confecção e adequação de órteses, comunicação alternativa e adequação postural em cadeiras de rodas. Essas ações ocuparam na média $52 \%$ da carga horária mensal dos fisioterapeutas, fonoaudiólogos e terapeutas ocupacionais, no período de 2000-2006. Os atendimentos foram realizados nas UBSs, na comunidade ou nos domicílios. As atividades em grupo se pautaram por diferentes modalidades: lúdicas, artísticas, corporais, educativas, terapêuticas, de auto cuidado, de lazer, oficinas etc. Os usuários que se encontravam restritos ao lar e necessitavam de adaptações das atividades de Vida Diária e Prática - AVD/AVP, adequação de mobiliário e ou equipamentos foram atendidos individualmente em visitas domiciliares.

- Construção de redes de apoio: uma das finalidades da construção de redes de apoio foi o desmonte dos processos de exclusão social das pessoas com incapacidades e deficiências. As situações de vulnerabilidade social como a segregação domiciliar e a exclusão dos equipamentos sociais, educativos e de trabalho foram problemas freqüentes em Sapopemba/Vila Prudente, a estratégia encontrada foi fazer parcerias os movimentos sociais de pessoas com deficiência do município e articular ações intersetoriais através de participações nos fóruns já existentes na região. Apenas 1,35\%, em média, da carga horária mensal dos profissionais de reabilitação, foram dedicados a essas atividades, no período entre 2000-2006, insuficiente para as necessidades detectadas, pois a articulação com outros setores fora da saúde são complexas e exigem tempo e dedicação.

- Ações educativas: compreende o conjunto de ações de promoção em saúde, prevenção e educação na comunidade, educação permanente com as equipes de saúde da família e equipes de apoio (saúde bucal, saúde mental e de reabilitação), oferta de estágios supervisionados para os estudantes da graduação em Fonoaudiologia da PUC de São Paulo e em Terapia Ocupacional da USP, além da formação individual dos profissionais de reabilitação em cursos de aprimoramento, especialização e mestrado. Essas atividades consumiram, em média, 10,5\% da carga horária mensal. É importante ressaltar que no primeiro ano de implantação da proposta, $18,4 \%$ da carga horária total dos profissionais de reabilitação foi utilizado em atividades de formação inicial para implantação do projeto. Nos cinco anos subseqüentes o tempo dedicado à educação permanente da equipe de reabilitação diminuiu para a média de $3,2 \%$. O tempo dedicado a ações dirigidas às outras equipes consistiu em $1,4 \%$ da carga horária mensal e de $3,2 \%$ para àquelas destinadas à comunidade.

- Reuniões Técnicas: abrange as reuniões com as equipes de saúde da família, saúde mental, saúde bucal, de especialistas e reuniões mensais da equipe de reabilitação. Os dois primeiros anos de implantação exigiram maior número e tempo em reuniões, cerca de $26 \%$ da carga horária mensal em 2001 e 32,5\% em 2002. Nos anos subseqüentes, com o estabelecimento de uma rotina intencional, no trabalho das equipes, o tempo foi reduzido para $19 \%$ da carga horária mensal.

- Trabalho administrativo: equivale ao horário para apontamentos nos prontuários, elaboração de relatórios e de projetos técnicos e registro dos procedimentos realizados. Os dois primeiros anos consumiram um tempo maior, $13,6 \%$ 
em 2001 e 13,2\% em 2002, reduzidos, em média, para $8,7 \%$ da carga horária mensal nos quatro anos seguintes. É importante ressaltar que esses registros contribuíram na organização das atividades de planejamento realizadas pelas equipes.

- Organização e planejamento das atividades: consiste no conjunto de reuniões realizadas a cada quadrimestre e/ou uma vez por ano para definir as ações específicas da equipe de reabilitação e as atividades compartilhadas com as outras equipes. Essa atividade só passou a ser registrada de forma discriminada em 2003, até então ficara indiferenciada nas reuniões técnicas e de trabalho administrativo. A média utilizada do tempo mensal foi de $4,6 \%$.

\section{5. criação de um banco de dados}

Foi criado em novembro de 2000 pela equipe de reabilitação da FZ um sistema de registro de informações das atividades desenvolvidas em forma de uma planilha de produção mensal de cada profissional. Essa planilha permitiu registrar as principais atividades e procedimentos realizados pela equipe de reabilitação e o faturamento do SIA/SUS. Além de apontar o tempo utilizado diariamente em cada atividade, a compilação desses dados permitiu orientar a organização da agenda mensal de cada profissional e de toda equipe de reabilitação, e proporcionou dados para avaliação da organização do trabalho e das metas propostas inicialmente.

\section{DISCUSSÃO: o modelo piramidal e a crítica de Capristano}

O projeto assistencial do SUS expresso na figura clássica da pirâmide (CECÍLIO, 1997) que representa o modelo tecno-assistencial desejado, divide as intervenções em atenção primária, secundária e terciária. Aqui as ações reabilitacionais estão inseridas no nível secundário e terciário. Consideramos que essa organização dos serviços não responde de forma efetiva aos problemas vivenciados cotidianamente pela maioria da população e dificultam o acesso e a integralidade nos cuidados em saúde.

Capistrano (1999) ao comentar a proposta da ESF/ FZ afirma que a diretriz primordial é a integração do atendimento ambulatorial básico de intercorrências clínicas, concomitantemente às ações de saúde pública, como as atividades educativas, vigilância sanitária, ações preventivas etc., preconizando, assim, a horizontalização da assistência. Lembra ainda que a AB/ESF pode buscar a integração de suas atividades com os hospitais de retaguarda e até organizar serviços híbridos como a Casa de Parto de Sapopemba (CAPISTRANO, 1999, p.93), portanto, os eixos norteadores da ESF/FZ seriam a ação em grupo, no território adstrito e a responsabilização integral por essa população.

$\mathrm{O}$ projeto de ações de reabilitação na $\mathrm{ESF} / \mathrm{FZ}$ ao assumir esses eixos norteadores previu intervenções consideradas, no modelo piramidal, como de nível secundário e terciário e produziu assim, um deslocamento epistemológico, organizacional e ético da prática profissional dos fisioterapeutas, fonoaudiólogos e terapeutas ocupacionais.

A inserção das ações de reabilitação na ESF demonstrou ser factível e eficaz, e demarcou novos princípios éticos e institucionais, sobretudo ao atingir pessoas com deficiências que em outras formas de organização de serviços ficam excluídas de qualquer intervenção, principalmente nos serviços de reabilitação especializados por tipo de deficiências.

Assim, a equipe de reabilitação passou a atender a partir da lógica da demanda da comunidade e não mais das especialidades como no modelo tradicional de reabilitação. A equipe de saúde da família tornou-se, assim, a porta de entrada para as ações de saúde e as ações reabilitacionais passaram a ser definidas pelas prioridades e condições de risco e vulnerabilidade da população e não mais por critérios de elegibilidade do serviço.

As equipes de saúde da família puderam, portanto, incorporar a pessoa com deficiência e/ou incapacidades em seus planos de trabalho e passaram a considerar as necessidades reabilitacionais como uma demanda importante e legítima da população, responsabilizando-se pela intervenção, como se pode observar nos documentos de planejamento das equipes de saúde da família.

Os ACSs, na medida em que se sentiram mais capacitados, em muitas ocasiões, desenvolveram ações junto à população com deficiências visando inseri-la nas práticas assistenciais da ESF e promover a qualidade de vida desses usuários no cotidiano domiciliar e da comunidade.

Neste modelo de intervenção, médicos, enfermeiros, dentistas começaram a diferenciar a ação reabilitacional clínica, de responsabilidade exclusiva da equipe de reabilitação, das ações gerais de saúde, de prevenção de incapacidades e de inserção social. Assim, a integralidade e a humanização dos serviços ganharam um novo matiz ético com a introdução das ações de reabilitação, pois efetiva o direito ao acesso irrestrito dos usuários.

Todavia, do ponto de vista da organização do trabalho em equipe, a proposta de interdisciplinaridade das ações não foi atingida de forma plena, muitas ações foram realizadas ainda a partir da formação específica dos profissionais, porém outras foram inseridas no contexto interdisciplinar.

O olhar sobre a problemática da pessoa com deficiência 
e/ou incapacidades também foi deslocado dos pressupostos organicistas que considera apenas o corpo patológico, para uma concepção que prevê o corpo relacional, inserido no espaço familiar e social, produzindo assim uma ruptura epistemológica com essa tradição tão arraigada no sistema de saúde. Assim, vários fatores, antes irrelevantes na configuração da intervenção, passaram a ser considerados. A intervenção deixou de ser a correção para se tornar o fortalecimento das potencialidades do sujeito, da família e da comunidade.

Se o objetivo primordial da intervenção reabilitacional tradicional está em minimizar ou eliminar a deficiência, na proposta da ESF a finalidade da reabilitação tornou-se o fortalecimento da autonomia e independência das pessoas com deficiências e de seus familiares, promovendo ações efetivas de cuidados em saúde e de participação social.

No espaço da ESF, as ações reabilitacionais foram organizadas de forma intrínseca com as atividades das equipes de saúde da família, que, são referência para o usuário, sua família e responsáveis. A equipe de reabilitação tornou-se, portanto, equipe matricial (CAMPOS; DOMITTI, 2007), e o seu objetivo foi assegurar a retaguarda especializada aos profissionais das equipes de saúde da família. A equipe matricial encarrega-se tanto de procedimentos especializados quanto de suporte técnico-pedagógico às equipes de referência. A organização das atividades de intervenção da equipe de reabilitação enquanto equipe matricial dependeu das diretrizes clínicas e sanitárias do território de referência, ou seja, não houve uma padronização a priori das atividades a serem desenvolvidas pelos profissionais de reabilitação. Assim, o que é oferecido em termos de ações reabilitacionais em uma UBS nem sempre é igual ao de outro equipamento de saúde, pois as características dos cuidados em saúde são dadas pelas necessidades da população local.

\section{ALGUMAS CONCLUSÕES}

A inserção das ações de reabilitação na ESF vem complementar a proposta de equipes de referência e equipes matriciais e responde a uma necessidade de saúde reprimida em função do modelo assistencial tradicional que não permite $\mathrm{o}$ acesso e a integralidade na assistência de pessoas com incapacidades e deficiências.

Os dados da pesquisa possibilitaram a compreensão da constituição das atividades de reabilitação na ESF e as atribuições dessas equipes matriciais. Demonstraram também como é importante estabelecer estratégias específicas que facilitem a incorporação das ações de reabilitação na ESF, com responsabilização por parte de todos os profissionais de saúde pelos usuários com deficiências e incapacidades.

A proposta das ações de reabilitação na $\mathrm{AB} / \mathrm{ESF}$ contribui, portanto, para a facilitação do acesso, da integralidade e humanização efetiva das ações em saúde. Redefine a reabilitação, que deixa de ser uma modalidade especializada dos serviços de saúde para ser parte das intervenções em saúde, garantindo à pessoa com deficiência o exercício pleno dos seus direitos como usuário do SUS e colocando as instituições de saúde a serviço de toda a população que compõe o território de cada UBS.

ROCHA, E. F.; KRETZER, M. R. Rehabilitation actions for disabled people in the strategy of family health of the Zerbini Foundation and the Municipal Health Department of São Paulo - Southeast Region - Sapopemba/Vila Prudente - period 2000/2006. Rev. Ter. Ocup. Univ. São Paulo, v. 20, n. 1, p. 59-67, jan./abr. 2009.

\begin{abstract}
S: The purpose of this article is to describe the implantation of rehabilitation actions in the Strategy of Family Health in the Region of Sapopemba/Vila Prudente - Zerbini Foundation/ Municipal Health Department of São Paulo, in the period of 2000-2006. The research sources were the documents recording the experience: the settlement project (2000), the evaluation reports (2001, 2003, 2008), notes of the rehabilitation staff meetings (2000-2006), the semiannual and annual plans, the daily activities summary tables of physiotherapy, phonoaudiology (speech therapy) and occupational therapy professionals and of the outpatiens treatment (2000-2006). As a result one can visualize: 1 . the strategies used to develop the proposal and the challenges faced; 2 . the routine established by the rehabilitation staff for the family health, mental health and oral health personnel; 3. the profile of the patient population and the criteria for eligibility; and 4. the types of assistance developed. The data made it possible to understand the establishing of the rehabilitation activities in the Strategy of Family Health, these prime groups chores and the ethical, epistemological and institutional rupture with the traditional rehabilitation model.
\end{abstract}

KEY WORDS: Family health. Rehabilitation. Primary health care. Physical therapy modalities. Speech therapy. Occupational therapy. 


\section{REFERÊNCIAS}

BRASIL. Ministério da Saúde. Coordenação de Atenção a Grupos Especiais. Programa de Atenção à Saúde da Pessoa Portadora de Deficiência. Atenção à pessoa portadora de deficiência no Sistema Único de Saúde: planejamento e organização dos serviços. Brasília: Secretaria de Assistência à Saúde, 1993.

BRASIL. Ministério da Saúde. Portaria 154 de 24 de janeiro de 2008 cria os NASFs - Núcleo de Atenção Integral à Saúde da Família.

BORGET, Ir. M. M. M. et al. Estratégia saúde da família: a experiência da equipe de reabilitação. São Paulo: Martinari, 2008.

CAMPOS, E. W. de; DOMITTI, A. C. Apoio matricial e equipe de referência: uma metodologia para gestão do trabalho interdisciplinar em saúde. Cad. Saúde Pública, Rio de Janeiro, v. 23, n. 2, p. 399-407, 2007.

CAPISTRANO FILHO, D. O Programa de Saúde da Família em São Paulo. Estudos Avançados, v. 13, n. 33, p. 89-99, 1999.

CECILIO, L. C. de O. Modelos tecno-assistenciais em saúde: da pirâmide ao círculo, uma possibilidade a ser explorada. Cad. Saúde
Pública, v. 13, n. 3, p. 469-478, 1997.

ROCHA, E. F.; PAULA, A. R. de; KRETZER, M. R. O estudo de prevalência de deficiências e incapacidades como instrumento de planejamento das atividades de atenção à saúde e reabilitação no Programa Saúde da Família. Rev. Ter. Ocup. Univ. São Paulo, v. 15, n. 1, p. 1-10, 2004.

ROCHA, E. F.; KRETZER, M. R. Avaliação da implantação das ações de reabilitação no Programa da Saúde da Família (PSF) da Fundação Zerbini e Secretaria Municipal de Saúde de São Paulo - Região Sudeste - Sapopemba/Vila Prudente - período 2000/2006. Relatório técnico - Projeto CNPq - Conselho Nacional de Desenvolvimento Científico e Tecnológico Edital MCT-CNPq / MS-DAB/SAS - No 49/2005 - Processo 202234/2005-7. Agosto de 2008. (mimeo).

SÃO PAULO (Estado). Secretaria de Estado da Saúde. Projeto implantação do Programa de Atenção à Saúde da Pessoa Portadora de Deficiência no Programa Saúde da Família - QUALIS. São Paulo: USP/ Fundação Zerbini/ Secretaria de Estado da Saúde, 2000. 